\title{
Neutral Higgs-boson pair production at hadron colliders: QCD corrections
}

\author{
S. Dawson \\ Physics Department, Brookhaven National Laboratory, Upton, New York 11973 \\ S. Dittmaier \\ Theory Division, CERN, CH-1211 Geneva 23, Switzerland \\ M. Spira \\ II. Institut für Theoretische Physik, Universität Hamburg, Luruper Chaussee 149, D-22761 Hamburg, Germany
}

(Received 8 May 1998; published 6 November 1998)

\begin{abstract}
Neutral Higgs-boson-pair production provides the possibility of studying the trilinear Higgs couplings at future high-energy colliders. We present the QCD corrections to the gluon-initiated processes in the limit of a heavy top quark in the loops and the Drell-Yan-like pair production of scalar and pseudoscalar Higgs particles. The $p p$ cross sections are discussed for CERN LHC energies within the standard model and its minimal supersymmetric extension. The QCD corrections are large, enhancing the total cross sections significantly. [S0556-2821(98)03719-9]

PACS number(s): 12.60.Fr, 12.38.Bx, 12.60.Jv, 14.80.Cp
\end{abstract}

\section{INTRODUCTION}

The Higgs mechanism is a cornerstone of the standard model (SM) and its supersymmetric extensions. Thus the search for Higgs bosons is one of the most important endeavors at future high-energy experiments. In the SM one Higgs doublet $\Phi$ has to be introduced in order to break the electroweak symmetry, leading to the existence of one elementary Higgs boson $H$ [1]. The scalar sector of the SM is uniquely fixed by the vacuum expectation value $v$ of the Higgs doublet and the mass $M_{H}$ of the physical Higgs boson [2]. Once a Higgs particle is found, it is necessary to investigate its properties in order to reconstruct the Higgs potential and to verify that it is indeed the SM Higgs boson. A first step in this direction is the measurement of the trilinear selfcouplings, which are uniquely specified by the scalar potential

$$
V=\lambda\left(\Phi^{\dagger} \Phi-\frac{v^{2}}{2}\right)^{2} .
$$

The parameter $\lambda$ defines the strength of the Higgs selfinteractions. In the SM it is given by $\lambda=M_{H}^{2} /\left(2 v^{2}\right)$. At the tree level, $\lambda$ can only be probed through multiple Higgsboson interactions, and there are, at present, no direct experimental limits on $\lambda$. In extensions of the SM, such as models with an extended scalar sector, with composite particles or with supersymmetric partners, the self-couplings of the Higgs boson may be significantly different from the SM predictions. The limits that may be obtained on the trilinear self-coupling of the Higgs boson at the CERN Large Hadron Collider (LHC) and the impact of QCD corrections represent a particular topic of this paper.

Since the minimal supersymmetric extension of the standard model (MSSM) requires the introduction of two Higgs doublets in order to preserve supersymmetry, there are five elementary Higgs particles, two $C P$-even $(h, H),{ }^{1}$ one $C P$-odd $(A)$, and two charged $\left(H^{ \pm}\right)$. This leads to a large variety of self-interactions among them. At lowest order all couplings and masses of the MSSM Higgs sector are fixed by two independent input parameters, which are generally chosen as $\tan \beta=v_{2} / v_{1}$, the ratio of the two vacuum expectation values $v_{1,2}$, and the pseudoscalar Higgs-boson mass $M_{A}$. The selfinteractions among the Higgs bosons (at lowest order) are given in terms of the electroweak gauge couplings, $\tan \beta$ and $M_{A}$, and may be quite different from the ones of the SM, which are governed by the parameter $\lambda$. Higher-order corrections to the MSSM Higgs sector turn out to be important owing to the large top-quark mass $m_{t}$ [3-5]. They increase the upper bound on the light Higgs scalar mass $M_{h}$ from the $Z$-boson mass $M_{Z}$ to about $130 \mathrm{GeV}$, along with altering the Higgs-boson self-couplings with contributions proportional to $G_{F} m_{t}^{4} / M_{Z}^{2}$.

Higgs-boson pairs can be produced by several mechanisms at hadron colliders: Higgs bremsstrahlung $W^{*} / Z^{*} \rightarrow \phi_{1} \phi_{2} W / Z \quad[6] ; \quad$ vector-boson fusion $W W, Z Z \rightarrow \phi_{1} \phi_{2}[7]$; associated production $Z^{*} \rightarrow A h, A H$; Higgs radiation off top and bottom quarks, $g g$, $q \bar{q} \rightarrow Q \bar{Q} \phi_{1} \phi_{2} \quad[8] ;$ gluon-gluon collisions $g g \rightarrow \phi_{1} \phi_{2}$ $[9,10]$. At the LHC; gluon fusion is the dominant source of Higgs-boson pairs, although in some regions of the MSSM parameter space, vector-boson fusion [11] can be important. Note, however, that $g g \rightarrow H A$ represents an exceptional case, since this channel is suppressed with respect to the DrellYan-like process $q \bar{q} \rightarrow Z^{*} \rightarrow H A$, so that it will be very difficult to separate the gluon-fusion process in this case experimentally. The gluon-fusion process $g g \rightarrow h A$, on the other hand, is competitive with the Drell-Yan-like process $q \bar{q}$ $\rightarrow Z^{*} \rightarrow h A$.

\footnotetext{
${ }^{1}$ We have taken care that no confusion can arise from using the same symbol $H$ for the SM and the heavy $C P$-even MSSM Higgs particle.
} 
In this paper we present the QCD corrections to the DrellYan-like production and the gluon-gluon collision processes. The gluon-fusion processes are, in the SM, mediated by triangle and box loops of top and bottom quarks; in the SM, the contributions of the bottom quark can always be neglected. In the MSSM the quark contributions will be suppressed if the quarks are heavier than $\sim 400 \mathrm{GeV}$, and, for small $\tan \beta$, the top-quark loops dominate the $g g$ cross sections. The QCD corrections to the gluon-fusion processes have been obtained in the limit of a heavy top quark by means of lowenergy theorems and also by explicitly expanding all relevant one- and two-loop diagrams. The results are expected to be valid for small $\tan \beta$ in the MSSM and below the $t \bar{t}$ threshold of the top-quark loops in both the MSSM and SM, since in this regime effects of a finite top-quark mass are expected to be small. In the case of single-Higgs-boson production, the same procedure reproduces the known exact result for the next-to-leading-order (NLO) cross section within $5 \%$, for Higgs-boson masses below $2 m_{t}[12]$. The considered QCD corrections are important in the process of extracting limits on the Higgs-boson self-couplings reliably.

The paper is organized as follows. In the next section the low-energy theorems for the interactions of gluons with light Higgs bosons will be reviewed and the relevant interactions in the heavy-quark limit will be constructed. In Sec. III the details of the calculation will be described, and in Sec. IV we present the results for the SM and MSSM Higgs bosons. In Sec. V we give some conclusions.

\section{LOW-ENERGY THEOREMS}

In the low-energy limit of vanishing Higgs fourmomentum, the Higgs-field operator acts as a constant field. In this limit it is possible to derive an effective Lagrangian for the interactions of the Higgs bosons with gauge bosons, which is valid for light Higgs bosons. This effective Lagrangian has been successfully used to compute the QCD corrections to a number of processes, in particular to singleHiggs-boson production from gluon fusion at the LHC $[13,14]$. In this case, the result of using the low-energy theorems has been shown to agree with the exact two-loop calculation to better than $10 \%$ even for $M_{H}$ as large as $1 \mathrm{TeV}$. This lends legitimacy to our use of the low-energy theorems to compute QCD corrections to multiple-Higgs-boson production via gluon fusion.

In the limit of vanishing Higgs four-momentum, the entire interaction of the scalar Higgs particles $H_{i}$ with a heavy quark $Q$ can be generated by the substitution

$$
m_{Q}^{0} \rightarrow m_{Q}^{0}+\eta_{Q}^{0} \sum_{i} g_{Q}^{i} H_{i}
$$

in the Lagrangian of a heavy quark of bare mass $m_{Q}^{0}$ [15], where $\eta_{Q}^{0}=m_{Q}^{0} / v$ denotes the bare SM Yukawa coupling, which must not be included in the substitution. The symbol $g_{Q}^{i}$ is the relative strength of the heavy-quark Yukawa coupling:

$$
\mathcal{L}_{\mathrm{Yuk}}=-\eta_{Q} \sum_{i} g_{Q}^{i} \bar{Q} Q H_{i}
$$

In the SM, we have $g_{Q}^{i}=1$. The expressions for $g_{Q}^{i}$ in the MSSM are given in Ref. [14]. At higher orders this substitution has to be performed for the unrenormalized parameters $[14,16]$. In the following we restrict our analysis to the top-quark contributions. At NLO the effective interaction between several scalar Higgs fields and gluons can be obtained from the radiatively corrected effective Lagrangian of gluon fields:

$$
\mathcal{L}_{g g}=-\frac{1}{4} G^{a \mu \nu} G_{\mu \nu}^{a}\left[1+\Pi_{g g}^{t}(0)\right],
$$

with $\Pi_{g g}^{t}(0)$ denoting the top-quark contribution to the unrenormalized gluon vacuum polarization at zero-momentum transfer. At two-loop order, we have

$$
\begin{aligned}
\Pi_{g g}^{t}(0)= & \frac{\alpha_{s}^{(5)}}{\pi} \Gamma(1+\epsilon)\left(\frac{4 \pi \mu^{2}}{\left(m_{t}^{0}\right)^{2}}\right)^{\epsilon}\left\{\frac{1}{6 \epsilon}+\frac{\alpha_{s}^{(5)}}{\pi} \Gamma(1+\epsilon)\right. \\
& \left.\times\left(\frac{4 \pi \mu^{2}}{\left(m_{t}^{0}\right)^{2}}\right)^{\epsilon}\left[\frac{1}{16 \epsilon}+\mathcal{O}\left(\epsilon^{0}\right)\right]+\mathcal{O}\left(\alpha_{s}^{2}\right)\right\},
\end{aligned}
$$

where the strong-coupling constant $\alpha_{s}^{(5)}$ includes five light flavors. This means that the top-quark contribution to the running of $\alpha_{s}$ has been subtracted at vanishing momentum transfer. Hereafter, we drop the superscript 5 on $\alpha_{s}$. Performing the substitution, Eq. (2), and renormalizing the bare top mass $m_{t}^{0}$ via

$$
m_{t}^{0}=m_{t}\left[1-\frac{\alpha_{s}}{\pi} \Gamma(1+\epsilon)\left(\frac{4 \pi \mu^{2}}{m_{t}^{2}}\right)^{\epsilon}\left(\frac{1}{\epsilon}+\frac{4}{3}\right)+\mathcal{O}\left(\alpha_{s}^{2}\right)\right],
$$

where $m_{t}$ denotes the pole mass, we end up with the NLO result

$$
\mathcal{L}_{H^{n} g g}=\frac{\alpha_{s}}{12 \pi} G^{a \mu \nu} G_{\mu \nu}^{a} \log \left[1+\sum_{i} g_{t}^{i} \frac{H_{i}}{v}\right]\left\{1+\frac{11}{4} \frac{\alpha_{s}}{\pi}\right\} .
$$

The interaction of even numbers of pseudoscalar Higgs bosons with gluons can be determined analogously from Eq. (5) by substituting [16]

$$
\left(m_{t}^{0}\right)^{2} \rightarrow\left(m_{l}^{0}\right)^{2}+\left(g_{t}^{A} \eta_{t}^{0} A\right)^{2},
$$

leading to

$$
\mathcal{L}_{A^{2 n} g g}=\frac{\alpha_{s}}{24 \pi} G^{a \mu \nu} G_{\mu \nu}^{a} \log \left[1+\left(g_{t}^{A} \frac{A}{v}\right)^{2}\right]\left\{1+\frac{11}{4} \frac{\alpha_{s}}{\pi}\right\} .
$$

The case of odd numbers of pseudoscalar Higgs bosons can be derived from the Adler-Bell-Jackiw (ABJ) anomaly [17] 

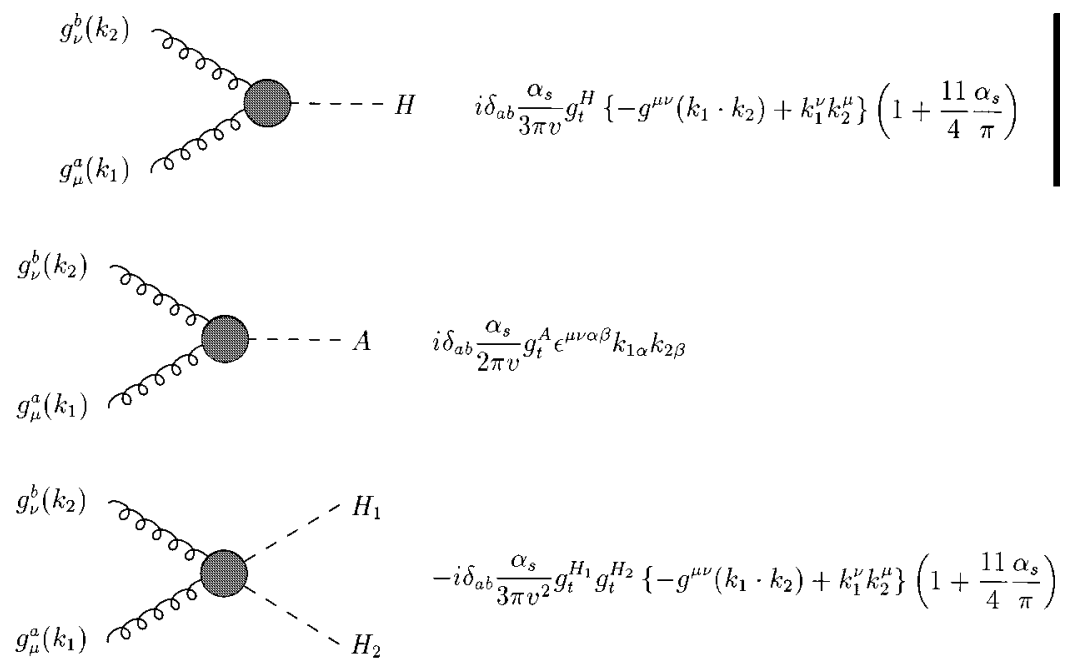

FIG. 1. Feynman rules for the effective interactions of Higgs bosons with gluons in the heavyquark limit, including NLO corrections.

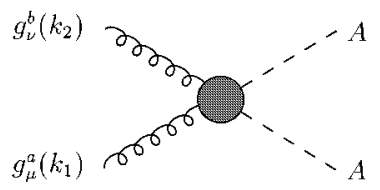

$$
i \delta_{a b} \frac{\alpha_{s}}{3 \pi v^{2}}\left(g_{i}^{A}\right)^{2}\left\{-g^{\mu \nu}\left(k_{1} \cdot k_{2}\right)+k_{1}^{\nu} k_{2}^{\mu}\right\}\left(1+\frac{11}{4} \frac{\alpha_{s}}{\pi}\right)
$$

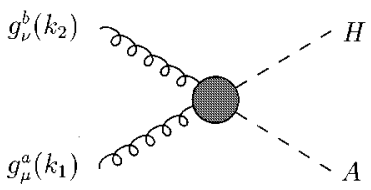

$-i \delta_{a b} \frac{\alpha_{s}}{2 \pi v^{2}} g_{t}^{H} g_{t}^{A} \epsilon^{\mu \nu \alpha \beta} k_{1 \alpha} k_{2 \beta}$

in the divergence of the axial-vector current $[14,16,18]$. The interactions that are relevant in our case are ${ }^{2}$

$$
\begin{gathered}
\mathcal{L}_{A g g}=g_{t}^{A} \frac{\alpha_{s}}{8 \pi} G^{a \mu \nu} \widetilde{G}_{\mu \nu}^{a} \frac{A}{v}, \\
\mathcal{L}_{A H g g}=-g_{t}^{A} g_{t}^{H} \frac{\alpha_{s}}{8 \pi} G^{a \mu \nu} \widetilde{G}_{\mu \nu}^{a} \frac{A H}{v^{2}},
\end{gathered}
$$

where $\widetilde{G}_{\mu \nu}^{a}=\frac{1}{2} \epsilon_{\mu \nu \alpha \beta} G^{a \alpha \beta}$ denotes the dual gluon-fieldstrength tensor. Owing to the Adler-Bardeen theorem [19], there are no higher-order corrections to the effective Lagrangians involving odd numbers of pseudoscalar Higgs bosons.

Figure 1 summarizes the Feynman rules for the effective interactions between two gluons and one or two Higgs bosons; the rules can be read off from Eqs. (7)-(10).

These Feynman rules can now be used to compute Higgs interactions beyond the lowest order. We recall that there is no contribution of light quarks (which are considered to be

${ }^{2}$ Note that in the earlier references $[14,16,18]$ a factor of $1 / 2$ is missing in the effective Lagrangians for the single pseudoscalar Higgs-boson coupling to gluons. massless) to the effective couplings, but note that light-quark loops have to be included when the Higgs bosons do not directly couple to the quark loops. Such contributions arise, in particular, in $g g \rightarrow Z^{*} \rightarrow h A, H A$ and cannot be obtained from the low-energy theorems.

\section{QCD CORRECTIONS}

\section{A. Gluon fusion: Basic definitions}

At leading order (LO) neutral-Higgs-boson-pair production via gluon fusion is mediated by triangle and box diagrams of heavy quarks, as exemplified in Fig. 2.

In the heavy-quark limit, the fermion triangles and boxes can be replaced by the effective vertices of Fig. 1. Throughout this analysis, we choose the squark masses to be $1 \mathrm{TeV}$ so that squark-loop contributions can be neglected in the MSSM case. Generically, the partonic LO cross section can be expressed as [10]
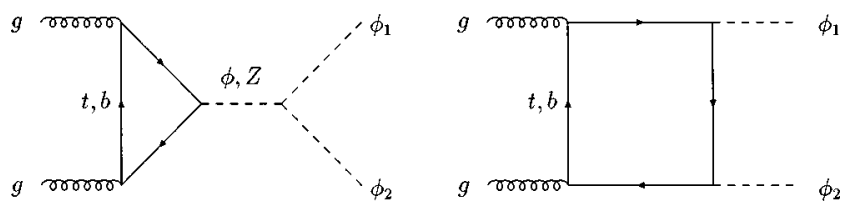

FIG. 2. Generic diagrams describing neutral Higgs-boson pair production in gluon-gluon collisions $\left(\phi, \phi_{i}=h, H, A\right)$. 


$$
\begin{aligned}
\hat{\sigma}_{\mathrm{LO}}(g g & \left.\rightarrow \phi_{1} \phi_{2}\right) \\
= & \int_{\hat{t}_{-}}^{\hat{t}_{+}} d \hat{t} \frac{G_{F}^{2} \alpha_{s}^{2}(\mu)}{256(2 \pi)^{3}}\left\{\left|C_{\triangle} F_{\triangle}+C_{\square} F_{\square}\right|^{2}\right. \\
& \left.+\left|C_{\square} G_{\square}\right|^{2}\right\} .
\end{aligned}
$$

The Mandelstam variables for the parton process are given by

$$
\begin{aligned}
& \hat{s}=Q^{2}, \\
& \hat{t}=-\frac{1}{2}\left[Q^{2}-m_{1}^{2}-m_{2}^{2}-\sqrt{\lambda\left(Q^{2}, m_{1}^{2}, m_{2}^{2}\right)} \cos \theta\right], \\
& \hat{u}=-\frac{1}{2}\left[Q^{2}-m_{1}^{2}-m_{2}^{2}+\sqrt{\lambda\left(Q^{2}, m_{1}^{2}, m_{2}^{2}\right)} \cos \theta\right],
\end{aligned}
$$

where $\theta$ is the scattering angle in the partonic c.m. system with invariant mass $Q$ and

$$
\lambda(x, y, z)=(x-y-z)^{2}-4 y z .
$$

The integration limits

$$
\hat{t}_{ \pm}=-\frac{1}{2}\left[Q^{2}-m_{1}^{2}-m_{2}^{2} \mp \sqrt{\lambda\left(Q^{2}, m_{1}^{2}, m_{2}^{2}\right)}\right]
$$

in Eq. (11) correspond to $\cos \theta= \pm 1$. The scale parameter $\mu$ is the renormalization scale. The complete dependence on the fermion masses is contained in the functions $F_{\triangle}, F_{\square}$, and $G_{\square}$. The full expressions of the form factors $F_{\triangle}, F_{\square}$, and $G_{\square}$, including the exact dependence on the fermion masses, can be found in Ref. [10].

The couplings $C_{\triangle}$ and $C_{\square}$ and the form factors $F_{\triangle}, F_{\square}$, and $G_{\square}$ in the heavy-quark limit are given by the following.

(i) $S M$ :

$$
\begin{aligned}
& C_{\triangle}=\lambda_{H H H} \frac{M_{Z}^{2}}{\hat{s}-M_{H}^{2}+i M_{H} \Gamma_{H}}, \quad C_{\square}=1, \\
& F_{\triangle} \rightarrow \frac{2}{3}, \quad F_{\square} \rightarrow-\frac{2}{3}, \\
& G_{\square} \rightarrow 0,
\end{aligned}
$$

with the trilinear coupling $\lambda_{H H H}=3 M_{H}^{2} / M_{Z}^{2}$.

(ii) MSSM: The couplings for the processes $g g$ $\rightarrow \phi_{1} \phi_{2}$ are generically defined as $\left(\phi, \phi_{i}=h, H, A\right)$

$$
C_{\Delta}^{\phi}=\lambda_{\phi_{1} \phi_{2} \phi} \frac{M_{Z}^{2}}{\hat{s}-M_{\phi}^{2}+i M_{\phi} \Gamma_{\phi}} g_{t}^{\phi}, \quad C_{\square}=g_{t}^{\phi_{1}} g_{t}^{\phi_{2}},
$$

where $\phi$ denotes the Higgs particles of the $s$-channel contributions. The trilinear couplings $\lambda_{\phi_{1} \phi_{2} \phi}$ and the normalized
Yukawa couplings $g_{t}^{\phi}$ can be found in Ref. [10]. The individual expressions in the heavy-quark limit can be summarized as follows.

$\phi_{1} \phi_{2}=h h, h H, H H$

$C_{\triangle}=C_{\triangle}^{h}+C_{\triangle}^{H}$,

$F_{\triangle} \rightarrow \frac{2}{3}, \quad F_{\square} \rightarrow-\frac{2}{3}$,

$G_{\square} \rightarrow 0$.

$\phi_{1} \phi_{2}=h A, H A:$

$C_{\triangle}=C_{\triangle}^{A}+C_{\triangle}^{Z}, \quad C_{\Delta}^{Z}=\lambda_{Z A h, Z A H} \frac{M_{Z}^{2}}{\hat{s}-M_{Z}^{2}+i M_{Z} \Gamma_{Z}} a_{t}$,

$F_{\triangle}^{A} \rightarrow 1, \quad F_{\triangle}^{Z} \rightarrow \frac{\hat{s}-M_{Z}^{2}}{M_{Z}^{2} \hat{s}}\left(M_{h, H}^{2}-M_{A}^{2}\right)$,

$F_{\square} \rightarrow-1, \quad G_{\square} \rightarrow 0$,

where $a_{t}=1$ denotes the axial charge of the top quark.

$\phi_{1} \phi_{2}=A A$ :

$$
\begin{aligned}
& C_{\triangle}=C_{\triangle}^{h}+C_{\triangle}^{H}, \\
& F_{\triangle} \rightarrow \frac{2}{3}, \quad F_{\square} \rightarrow \frac{2}{3}, \\
& G_{\square} \rightarrow 0 .
\end{aligned}
$$

It should be noted that owing to the Ward identities for the $Z g g$ vertex only the pseudoscalar Goldstone component of the $Z$ bosons contributes to $F_{\triangle}^{Z}$ in the case of $h A$ and $H A$ production in the heavy-quark limit. The anomaly contributions of the top and bottom quarks cancel.

The QCD corrections consist of two-loop virtual corrections, generated by gluon exchange between the quark lines and/or external gluons, and one-loop real corrections with an additional gluon or light quark in the final state. We have evaluated the QCD corrections in the heavy-quark limit by means of two different methods: (i) using the effective couplings based on the low-energy theorems, as presented in the previous section, and (ii) explicitly expanding all relevant

(a)
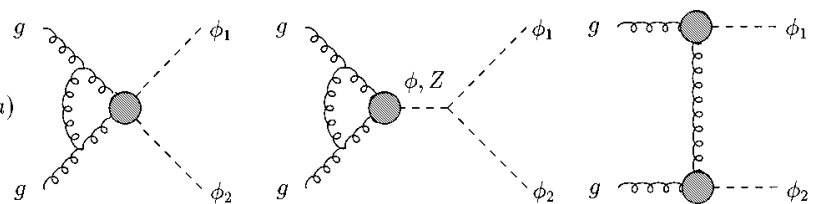

(b)
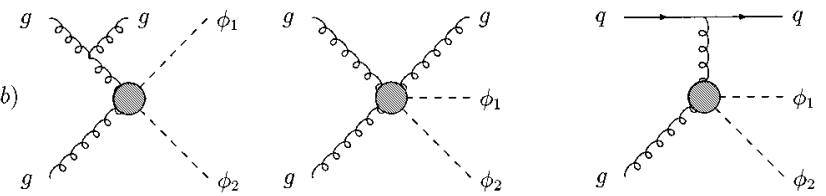

FIG. 3. Typical effective diagrams contributing to the (a) virtual and (b) real corrections to neutral Higgs-boson-pair production. 
one- and two-loop diagrams in the inverse heavy-quark mass. In the following we shall describe the details of both approaches.

\section{B. Low-energy theorems}

We are now in a position to compute the NLO corrections to Higgs-boson pair production. Typical effective diagrams contributing to the virtual and real corrections are presented in Fig. 3.

Adopting the Feynman rules of Fig. 1 for the effective interactions, the calculation has been carried out in dimensional regularization with $n=4-2 \epsilon$ dimensions. The strong coupling has been renormalized in the modified minimal subtraction $(\overline{\mathrm{MS}})$ scheme including five light-quark flavors, i.e., decoupling the top quark in the running of $\alpha_{s}$. After summing the virtual and real corrections, the infrared singularities cancel. However, collinear initial-state singularities are left over in the partonic cross sections. Those divergences have been absorbed into the NLO parton densities, defined in the $\overline{\mathrm{MS}}$ scheme with five light-quark flavors. We end up with finite results, which can be cast into the form

$$
\begin{aligned}
\sigma_{\mathrm{NLO}}(p p & \left.\rightarrow \phi_{1} \phi_{2}+X\right) \\
& =\sigma_{\mathrm{LO}}+\Delta \sigma_{\mathrm{virt}}+\Delta \sigma_{g g}+\Delta \sigma_{g q}+\Delta \sigma_{q \bar{q}},
\end{aligned}
$$

with the individual contributions

$$
\begin{aligned}
& \sigma_{\mathrm{LO}}=\int_{\tau_{0}}^{1} d \tau \frac{d \mathcal{L}^{g g}}{d \tau} \hat{\sigma}_{\mathrm{LO}}\left(Q^{2}=\tau s\right) \\
& \Delta \sigma_{\text {virt }}=\frac{\alpha_{s}(\mu)}{\pi} \int_{\tau_{0}}^{1} d \tau \frac{d \mathcal{L}^{g g}}{d \tau} \hat{\sigma}_{\mathrm{LO}}\left(Q^{2}=\tau s\right) C, \\
& \Delta \sigma_{g g}=\frac{\alpha_{s}(\mu)}{\pi} \int_{\tau_{0}}^{1} d \tau \frac{d \mathcal{L}^{g g}}{d \tau} \int_{\tau_{0} / \tau}^{1} \frac{d z}{z} \hat{\sigma}_{\mathrm{LO}}\left(Q^{2}=z \tau s\right)\left\{-z P_{g g}(z) \log \frac{M^{2}}{\tau s}-\frac{11}{2}(1-z)^{3}+6\left[1+z^{4}+(1-z)^{4}\right]\left(\frac{\log (1-z)}{1-z}\right)+\right. \\
& \Delta \sigma_{g q}=\frac{\alpha_{s}(\mu)}{\pi} \int_{\tau_{0}}^{1} d \tau \sum_{q, q} \frac{d \mathcal{L}^{g q}}{d \tau} \int_{\tau_{0} / \tau}^{1} \frac{d z}{z} \hat{\sigma}_{\mathrm{LO}}\left(Q^{2}=z \tau s\right)\left\{-\frac{z}{2} P_{g q}(z) \log \frac{M^{2}}{\tau s(1-z)^{2}}+\frac{2}{3} z^{2}-(1-z)^{2}\right\}, \\
& \Delta \sigma_{q \bar{q}}=\frac{\alpha_{s}(\mu)}{\pi} \int_{\tau_{0}}^{1} d \tau \sum_{q} \frac{d \mathcal{L}^{q \bar{q}}}{d \tau} \int_{\tau_{0} / \tau}^{1} \frac{d z}{z} \hat{\sigma}_{\mathrm{LO}}\left(Q^{2}=z \tau s\right) \frac{32}{27}(1-z)^{3} .
\end{aligned}
$$

The coefficient $C$ for the virtual corrections reads

$$
C=\pi^{2}+c_{1}+\frac{33-2 N_{F}}{6} \log \frac{\mu^{2}}{Q^{2}}+\operatorname{Re} \frac{\int_{\tilde{t}_{-}}^{\tilde{t}_{+}} d \hat{t}\left\{c_{2} C_{\square}\left(C_{\triangle} F_{\triangle}+C_{\square} F_{\square}\right)+c_{3}\left(p_{T}^{2} / 2 \hat{t} \hat{u}\right)\left(Q^{2}-m_{1}^{2}-m_{2}^{2}\right) C_{\square}^{2} G_{\square}\right\}}{\int_{\tilde{t}_{-}}^{\tilde{t}_{+}} d \hat{t}\left\{\left|C_{\triangle} F_{\triangle}+C_{\square} F_{\square}\right|^{2}+\left|C_{\square} G_{\square}\right|^{2}\right\}},
$$

where

$$
\tau_{0}=\frac{\left(m_{1}+m_{2}\right)^{2}}{s}, \quad p_{T}^{2}=\frac{\left(\hat{t}-m_{1}^{2}\right)\left(\hat{u}-m_{1}^{2}\right)}{Q^{2}}-m_{1}^{2} .
$$

The objects $P_{g g}(z)$ and $P_{g q}(z)$ denote the Altarelli-Parisi splitting functions [20]:

$$
\begin{aligned}
P_{g g}(z)= & 6\left\{\left(\frac{1}{1-z}\right)+\frac{1}{z}-2+z(1-z)\right\} \\
& +\frac{33-2 N_{F}}{6} \delta(1-z), \\
P_{g q}(z)= & \frac{4}{3} \frac{1+(1-z)^{2}}{z},
\end{aligned}
$$

where $N_{F}=5$ in our case. The factorization scale of the parton-parton luminosities $d \mathcal{L}^{i j} / d \tau$ is denoted by $M$. The coefficients $c_{i}$ for the individual final-state Higgs bosons $\phi_{1} \phi_{2}$ are given by

$\phi_{1} \phi_{2}=h h, h H, H H: \quad c_{1}=\frac{11}{2}, \quad c_{2}=\frac{4}{9}, \quad c_{3}=-\frac{4}{9}$;

$\phi_{1} \phi_{2}=h A, H A: \quad c_{1}=6, \quad c_{2}=\frac{2}{3}, \quad c_{3}=\frac{2}{3} \frac{\hat{t}-\hat{u}}{Q^{2}-m_{1}^{2}-m_{2}^{2}}$

$\phi_{1} \phi_{2}=A A: \quad c_{1}=\frac{11}{2}, \quad c_{2}=-1, \quad c_{3}=-1$.

In order to improve the validity of our results, we have inserted the full expressions for the form factors $F_{\triangle}, F_{\square}$, and 
$G_{\square}$ in Eqs. (11) and (22), i.e., including the exact dependence on the fermion masses. This procedure is reasonable since the QCD corrections are dominated by soft and collinear gluon radiation, which do not resolve the Higgs couplings to gluons, analogously to single-Higgs-boson production via gluon fusion [12].

A few remarks on the $s$-channel $Z$-boson exchange in $h A, H A$ production are in order. For the virtual corrections, the factorization of the NLO corrections into the LO form factors and a universal correction factor is exact for $m_{t} \rightarrow \infty$ and $m_{b}=0$. This is due to the fact that only the pseudoscalar Goldstone component of the $Z$ boson contributes as in LO; i.e., the QCD corrections coincide with the one to $s$-channel pseudoscalar Higgs-boson exchange. For the real corrections, the factorization is not exact, but the applied correction factor correctly includes the dominant contributions, which are caused by soft and collinear gluon radiation. Additional infrared- and collinear-finite contributions, e.g., originating from Zggg box corrections in $g g \rightarrow g+Z^{*} \rightarrow g+h A, H A$ processes, are expected to be small, since they do not exhibit large contributions from soft and collinear gluon radiation. They are neglected in our analysis.

\section{Explicit expansion of the gluon-fusion diagrams}

We have derived the above results also by explicitly performing the heavy-mass expansion of the corresponding oneand two-loop Feynman diagrams. The amplitudes for the individual diagrams have been automatically generated using the package FEYNARTS [21]. The asymptotic expansion of the individual amplitudes in the heavy top-quark mass is carried out directly in the integrand, i.e., before the integration over the momentum space. We employ the general algorithm of Ref. [22] (see also Ref. [23] and references therein) for the asymptotic expansion of Feynman diagrams in dimensional regularization. This method expresses the coefficients of the expansion in terms of simpler diagrams. At the one-loop level, this procedure leads to simple one-loop vacuum integrals only. At the two-loop level, we get two-loop vacuum integrals and products of one-loop vacuum integrals and massless one-loop integrals with nonvanishing external momenta. The analytical calculation of all those integrals is straightforward when using the Feynman-parameter technique. Since the employed strategy leads to a very large number of terms in intermediate steps and since each step is algorithmic, we have fully automatized the calculation in MATHEMATICA [24]. In the following we sketch the single steps of the calculation and give the results for the basic integrals.

The general algorithm for the asymptotic expansion of any given Feynman graph $\Gamma$ in the limit $M_{i} \rightarrow \infty$ for some internal masses $M_{i}$ can easily be summarized. Denoting the corresponding Feynman amplitude by $F_{\Gamma}$ and the corresponding integrand by $I_{\Gamma}$, the large-mass expansion reads

$$
\begin{aligned}
F_{\Gamma}= & \int\left(\prod_{l} d^{n} q_{l}\right) \\
& \times I_{\Gamma} \underset{M_{i} \rightarrow \infty}{\sim} \sum_{\gamma} \int\left(\prod_{l} d^{n} q_{l}\right) I_{\Gamma / \gamma} \mathcal{T}_{p_{i}^{\gamma}, m_{i}} I_{\gamma},
\end{aligned}
$$

where $q_{l}$ are the integration momenta. The sum on the righthand side runs over all subgraphs $\gamma$ of $\Gamma$ which contain all propagators with the heavy masses $M_{i}$ and which are irreducible with respect to those lines of $\gamma$ that carry light masses $m_{i}$. The integrand of the subgraph $\gamma$ is denoted by $I_{\gamma}$. The reduced graph $\Gamma / \gamma$ results from $\Gamma$ upon shrinking $\gamma$ to a point, and the integrand $I_{\Gamma / \gamma}$ is defined such that $I_{\Gamma}$ $=I_{\gamma} I_{\Gamma / \gamma}$. The symbol $\mathcal{T}_{p_{i}^{\gamma}, m_{i}}$ represents an operator that replaces the integrand $I_{\gamma}$ by its Taylor series in the expansion parameters $p_{i}^{\gamma}$ and $m_{i}$, where $p_{i}^{\gamma}$ are the external momenta of the subgraph $\gamma$. Therefore, Eq. (26) expresses the original integral $F_{\Gamma}$ in terms of an infinite sum over simpler integrals. For any given power $\xi^{a}$, this sum contains only a finite number of terms that are nonvanishing in $\left(F_{\Gamma \mid M_{i} \rightarrow \xi M_{i}}\right) / \xi^{a}$ after the scaling limit $\xi \rightarrow \infty$ is taken. These terms can easily be determined by power counting. This general strategy for the heavy-mass expansion will become more transparent when we inspect in more detail the different types of graphs that are relevant in our case.

We start by considering the relevant one-loop integrals in the limit $m_{t} \rightarrow \infty$. They contain only top-quark propagators in the loop, both for the LO calculation and for the real NLO corrections. According to the algorithm (26), there is only one relevant subgraph $\gamma$, namely, the subgraph $\gamma_{\text {loop }}$ containing only the propagators of lines inside the loop. If $\Gamma$ is irreducible, we have $\Gamma=\gamma_{\text {loop }}$. The Taylor-expansion operator $\mathcal{T}$ replaces each propagator $P\left(q-p, m_{t}\right)$ by

$$
\begin{aligned}
P\left(q-p, m_{t}\right) & =\left[(q-p)^{2}-m_{t}^{2}\right]^{-1} \\
& =\sum_{l=0}^{\infty}\left(q^{2}-m_{t}^{2}\right)^{-1-l}\left(2 q p-p^{2}\right)^{l},
\end{aligned}
$$

where $q$ denotes the integration momentum and $p$ is any combination of external momenta. These replacements express each one-loop diagram by a sum of terms containing one-loop vacuum integrals.

$$
V_{\mu_{1} \cdots \mu_{R}}^{(1)}\left(n_{1} ; m_{1}\right)=\frac{(2 \pi \mu)^{4-n}}{i \pi^{2}} \int d^{n} q \frac{q_{\mu_{1}} \cdots q_{\mu_{R}}}{\left(q^{2}-m_{1}^{2}\right)^{n_{1}}} .
$$

The terms that are nonvanishing in the heavy-mass limit can be determined by simple power counting, since an explicit factor of $m_{t}$ and the integration momentum $q$ contribute to the scaling factor in $m_{t}$ exactly in the same way. All nonvanishing vacuum tensor integrals (28) can be decomposed into terms that are products of metric tensors $g_{\mu \nu}$ and coefficient factors. The coefficients for the different covariants, which 


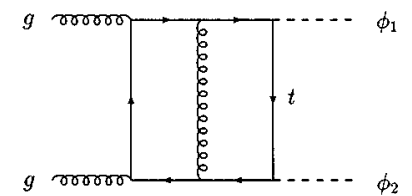

(a)

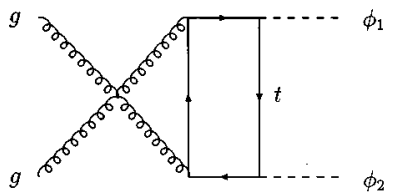

(c)

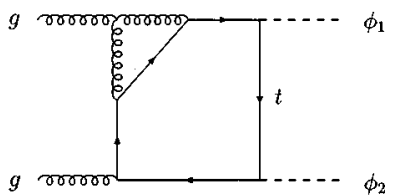

(b)

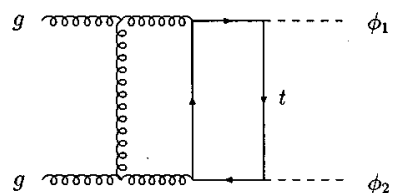

(d)
FIG. 4. Typical two-loop box diagrams with one, two, or three internal gluons.

span the whole tensor, can be algebraically expressed in terms of scalar vacuum integrals. This algebraic reduction, which proceeds recursively in the tensor rank, is standard. The trick is to contract the equation that expresses the integral in terms of covariants with a set of some suitably chosen covariants. On the side of the integral, this leads to integrals that are already known; on the other side of this equation, one gets linear combinations for the tensor coefficients. The coefficients are obtained by inverting a system of such linear equations for the coefficients. For the case of one-loop tensor vacuum integrals, at most a single covariant structure contributes, namely, the totally symmetric tensor built of metric tensors $g_{\mu \nu}$. Only scalar one-loop integrals, i.e., the ones of Eq. (28) with $R=0$, have to be computed explicitly. A simple calculation yields

$$
V^{(1)}\left(n_{1} ; m_{1}\right)=(-1)^{n_{1}}\left(4 \pi \mu^{2}\right)^{(4-n) / 2} m^{n-2 n_{1}} \frac{\Gamma\left(n_{1}-n / 2\right)}{\Gamma\left(n_{1}\right)} \text {. }
$$

At the two-loop level, there are two basically different types of diagrams. The first type contains two independent top-quark loops. Such diagrams do not lead to genuine twoloop integrals and can be treated like the one-loop diagrams above. Topologically, those diagrams are represented by the third graph of Fig. 3. The second type of graph is formed by the genuine two-loop diagrams. Each of those diagrams contains a closed top-quark loop and one, two, or three internal gluon lines. Typical box diagrams are shown in Fig. 4.

For all such genuine two-loop diagrams, there are two subgraphs $\gamma$ that are relevant in the expansion (26) for $m_{t}$ $\rightarrow \infty$. The first subgraph is the diagram $\gamma_{\text {loop }}$ built of all lines inside the loops: the second is given by the closed top-quark loop.

First, we consider the case $\gamma=\gamma_{\text {loop }}$. The Taylor expansion of the integrand $I_{\gamma_{\text {loop }}}$ involves the consistent expansion of each propagator about the external momenta of the process. This means that each propagator $P(q-p, m)$ is replaced by

$$
P(q-p, m)=\left[(q-p)^{2}-m^{2}\right]^{-1}
$$

$$
=\sum_{l=0}^{\infty}\left(q^{2}-m^{2}\right)^{-1-l}\left(2 q p-p^{2}\right)^{l}
$$

$$
m=0, m_{t},
$$

where $q$ is a linear combination of the two integration momenta $q_{1}$ and $q_{2}$, and $p$ consists of external momenta $p_{i}$. This replacement leads to two-loop vacuum integrals of the general form

$$
\begin{aligned}
& V_{\mu_{1} \cdots \mu_{R} ; \nu_{1} \cdots \nu_{S}}^{(2)}\left(n_{1}, n_{2}, n_{3} ; m_{1}, m_{2}, m_{3}\right) \\
& =\frac{(2 \pi \mu)^{4-n}}{i \pi^{2}} \int d^{n} q_{1} \frac{(2 \pi \mu)^{4-n}}{i \pi^{2}} \\
& \quad \times \int d^{n} q_{2} \frac{q_{1, \mu_{1}} \cdots q_{1, \mu_{R}} q_{2, \nu_{1}} \cdots q_{2, \nu_{S}}}{\left(q_{1}^{2}-m_{1}^{2}\right)^{n_{1}}\left(q_{2}^{2}-m_{2}^{2}\right)^{n_{2}}\left[\left(q_{1}+q_{2}\right)^{2}-m_{3}^{2}\right]^{n_{3}}}
\end{aligned}
$$

with the actual mass insertions $m_{1}=0$ and $m_{2}=m_{3}=m_{t}$. In the power counting, which determines the nonvanishing terms for $m_{t} \rightarrow \infty$, factors of the integration momenta $q_{1}$ and $q_{2}$ contribute in the same way as explicit factors of $m_{t}$. The algebraic reduction of two-loop tensor vacuum integrals proceeds along the same lines as described for the one-loop case above. The only difference is that tensors need not be totally symmetric beyond one loop. Finally, we are left with the scalar integral $(R=S=0)$, which can be easily calculated:

$$
\begin{aligned}
& V^{(2)}\left(n_{1}, n_{2}, n_{3} ; 0, m, m\right)=(-1)^{n_{1}+n_{2}+n_{3}}\left(4 \pi \mu^{2}\right)^{4-n}\left(m^{2}\right)^{n-n_{1}-n_{2}-n_{3}} \\
& \times \frac{\Gamma\left(n_{1}+n_{2}+n_{3}-n\right) \Gamma\left(n / 2-n_{1}\right) \Gamma\left(n_{1}+n_{2}-n / 2\right) \Gamma\left(n_{1}+n_{3}-n / 2\right)}{\Gamma(n / 2) \Gamma\left(n_{2}\right) \Gamma\left(n_{3}\right) \Gamma\left(2 n_{1}+n_{2}+n_{3}-n\right)} .
\end{aligned}
$$


Now we identify the subgraph $\gamma$ with the top-quark loop. In this case the Taylor expansion in the integrand $I_{\gamma}$ concerns the momenta that are external with respect to the topquark loop. Thus all top-quark propagators $P\left(q_{2}-p, m_{t}\right)$ are replaced by

$$
\begin{aligned}
P\left(q_{2}-p, m_{t}\right) & =\left[\left(q_{2}-p\right)^{2}-m_{t}^{2}\right]^{-1} \\
& =\sum_{l=0}^{\infty}\left(q_{2}^{2}-m_{t}^{2}\right)^{-1-l}\left(2 q_{2} p-p^{2}\right)^{l},
\end{aligned}
$$

where $q_{2}$ is the loop momentum running through the topquark loop. Note that $p$ includes all external momenta of the process as well as the loop momentum $q_{1}$ running through the internal gluon lines. The integration over $q_{2}$ leads to one-loop vacuum integrals of the form (28), the calculation of which is described above. The integration over $q_{1}$ involves only massless propagators to the first power. Since the $q_{1}$ integration does not involve any $m_{t}$ terms, it does not affect the power counting in $m_{t}$ at all. For one-gluon ex- change the integral over $q_{1}$ is a massless tadpole, which vanishes in dimensional regularization. For two- and threegluon exchange, the $q_{1}$ integration leads to the one-loop tensor integrals

$$
\begin{aligned}
B_{\mu_{1} \cdots \mu_{R}}(p)= & \frac{(2 \pi \mu)^{4-n}}{i \pi^{2}} \int d^{n} q_{1} \frac{q_{1, \mu_{1}} \cdots q_{1, \mu_{R}}}{q_{1}^{2}\left(q_{1}+p\right)^{2}}, \\
C_{\mu_{1} \cdots \mu_{R}}\left(p_{1}, p_{2}\right)= & \frac{(2 \pi \mu)^{4-n}}{i \pi^{2}} \\
& \times \int d^{n} q_{1} \frac{q_{1, \mu_{1}} \cdots q_{1, \mu_{R}}}{q_{1}^{2}\left(q_{1}+p_{1}\right)^{2}\left(q_{1}+p_{2}\right)^{2}} .
\end{aligned}
$$

The tensor integrals can again be recursively reduced to the corresponding scalar integrals in a fully algebraic manner. The relevant scalar integrals can be easily calculated and are given by

$$
\begin{gathered}
\left.B(p)\right|_{p^{2} \neq 0}=\left(\frac{4 \pi \mu^{2}}{-p^{2}-i 0}\right)^{(4-n) / 2} \frac{\Gamma(2-n / 2) \Gamma(n / 2-1)^{2}}{\Gamma(n-2)}, \\
\left.C\left(p_{1}, p_{2}\right)\right|_{p_{1}^{2}=p_{2}^{2}=0, p^{2}=\left(p_{1}-p_{2}\right)^{2} \neq 0}=\frac{1}{p^{2}}\left(\frac{4 \pi \mu^{2}}{-p^{2}-i 0}\right)^{(4-n) / 2} \frac{\Gamma(3-n / 2) \Gamma(n / 2-2)^{2}}{\Gamma(n-3)} .
\end{gathered}
$$

For $p^{2}=0$ the integrals are zero in dimensional regularization. The case $B(p)$ with $p^{2}=0$ occurs, for instance, in graphs like Fig. 4(b). Therefore we find that the contribution in the expansion (26) for which $\gamma$ is the top-quark loop is only nonvanishing in diagrams like Figs. 4(c) and 4(d), where both external gluons are attached to the internal gluon lines.

A few more "physical" remarks on the formally described algorithm for the asymptotic expansion seem to be in order. The different contributions to a given Feynman graph that are associated with the subgraphs $\gamma$ in the expansion (26) are directly related to the effective diagrams in the approach of the low-energy theorem described above. Shrinking the subgraph $\gamma$ to a point leads to the corresponding effective diagram, where the point arising from $\gamma$ is the pointlike interaction of the effective Lagrangian. A nonvanishing contribution of a subgraph $\gamma \neq \gamma_{\text {loop }}$ requires that at least one external momentum $p_{i}$ pass through a massless propagator; otherwise, the loop integral involving the massless propagators is zero. In other words, it is necessary that there exist a cut through the diagram that passes exclusively massless lines. Therefore only diagrams with such a "massless cut" can lead to contributions to effective diagrams in which an effective coupling appears in loops. But all diagrams in general contribute to treelike effective diagrams, which result from shrinking the complete loop part $\gamma_{\text {loop }}$ to a point.
Finally, we mention that $\gamma_{5}$, which appears in the case of pseudoscalar Higgs bosons, is treated according to the prescription of 't Hooft and Veltman [25]. Technically, we substitute $\gamma_{5}$ by $i \epsilon^{\mu_{1} \mu_{2} \mu_{3} \mu_{4}} \gamma_{\mu_{1}} \gamma_{\mu_{2}} \gamma_{\mu_{3}} \gamma_{\mu_{4}} / 4$ ! before the evaluation of the Dirac trace so that the actual trace calculation can be carried out for usual $n$-dimensional Dirac matrices. The correct projection of the trace result on the physical fourdimensional space is achieved upon the contraction with the four-dimensional $\epsilon$ tensor. In this approach, all loop integrations can be carried out before the contraction with $\epsilon$; i.e., $n$-dimensional momenta can be used. Note, however, that the contraction with $\epsilon$ necessarily occurs before the integration over the phase space of the radiated parton in the real corrections; i.e., one has to take care of the four-dimensionality of $\epsilon$ there. Moreover, an additional spurious counterterm has to be added to the $A t \bar{t}$ vertex (see also $[14,18]$ ).

\section{Drell-Yan-like processes}

Pairs of scalar and pseudoscalar Higgs bosons can also be produced in $q \bar{q}$ collisions via $s$-channel $Z$-boson exchange; see Fig. 5. At LO the partonic cross sections are given by

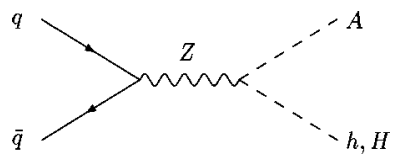

FIG. 5. Diagram contributing to $q \bar{q} \rightarrow A h, A H$ at lowest order. 


$$
\begin{aligned}
\hat{\sigma}_{\mathrm{LO}}(q \bar{q} & \rightarrow A h, A H) \\
& =\lambda_{Z A h, Z A H}^{2} \frac{G_{F}^{2} M_{Z}^{4}}{288 \pi}\left(v_{q}^{2}+a_{q}^{2}\right) \frac{\lambda\left(Q^{2}, M_{A}^{2}, M_{h, H}^{2}\right)^{3 / 2}}{\left(Q^{2}\right)^{2}\left(Q^{2}-M_{Z}^{2}\right)^{2}},
\end{aligned}
$$

where $Q^{2}$ denotes the partonic c.m. energy squared and $v_{q}$ $=2 I_{3 q}-4 e_{q} \sin ^{2} \theta_{W}, a_{q}=2 I_{3 q}$ are the vectorial and axial charges of the initial-state quarks. The QCD corrections coincide analytically with the QCD corrections to the DrellYan process $q \bar{q} \rightarrow Z^{*}$, if squarks and gluinos are heavy, so that their contributions can be neglected. Thus the NLO cross section can be expressed as

$$
\begin{aligned}
\sigma_{\mathrm{NLO}}(p p \rightarrow & A h, A H+X) \\
= & \sigma_{\mathrm{LO}}+\Delta \sigma_{q \bar{q}}+\Delta \sigma_{g g}, \\
\sigma_{\mathrm{LO}}= & \int_{\tau_{0}}^{1} d \tau \sum_{q} \frac{d \mathcal{L}^{q \bar{q}}}{d \tau} \hat{\sigma}_{\mathrm{LO}}\left(Q^{2}=\tau s\right), \\
\Delta \sigma_{q \bar{q}}= & \frac{\alpha_{s}(\mu)}{\pi} \int_{\tau_{0}}^{1} d \tau \sum_{q} \frac{d \mathcal{L}^{q \bar{q}}}{d \tau} \\
& \times \int_{\tau_{0} / \tau}^{1} d z \hat{\sigma}_{\mathrm{LO}}\left(Q^{2}=\tau z s\right) \omega_{q \bar{q}}(z), \\
\Delta \sigma_{q g}= & \frac{\alpha_{s}(\mu)}{\pi} \int_{\tau_{0}}^{1} d \tau \sum_{q, \bar{q}} \frac{d \mathcal{L}^{q g}}{d \tau} \\
& \times \int_{\tau_{0} / \tau}^{1} d z \hat{\sigma}_{\mathrm{LO}}\left(Q^{2}=\tau z s\right) \omega_{q g}(z),
\end{aligned}
$$

with the coefficient functions [26]

$$
\begin{aligned}
\omega_{q \bar{q}}(z)= & -P_{q q}(z) \log \frac{M^{2}}{\tau S}+\frac{4}{3}\left\{2\left[\zeta_{2}-2\right] \delta(1-z)\right. \\
& \left.+4\left(\frac{\log (1-z)}{1-z}\right)_{+}-2(1+z) \log (1-z)\right\}, \\
\omega_{q g}(z)= & -\frac{1}{2} P_{q g}(z) \log \left(\frac{M^{2}}{(1-z)^{2} \tau S}\right) \\
& +\frac{1}{8}\left\{1+6 z-7 z^{2}\right\} .
\end{aligned}
$$

The Altarelli-Parisi splitting functions $P_{q \bar{q}}$ and $P_{q g}$ are given by [20]

$$
\begin{aligned}
& P_{q q}(z)=\frac{4}{3}\left\{2\left(\frac{1}{1-z}\right)+1-z+\frac{3}{2} \delta(1-z)\right\}, \\
& P_{q g}(z)=\frac{1}{2}\left\{z^{2}+(1-z)^{2}\right\} .
\end{aligned}
$$

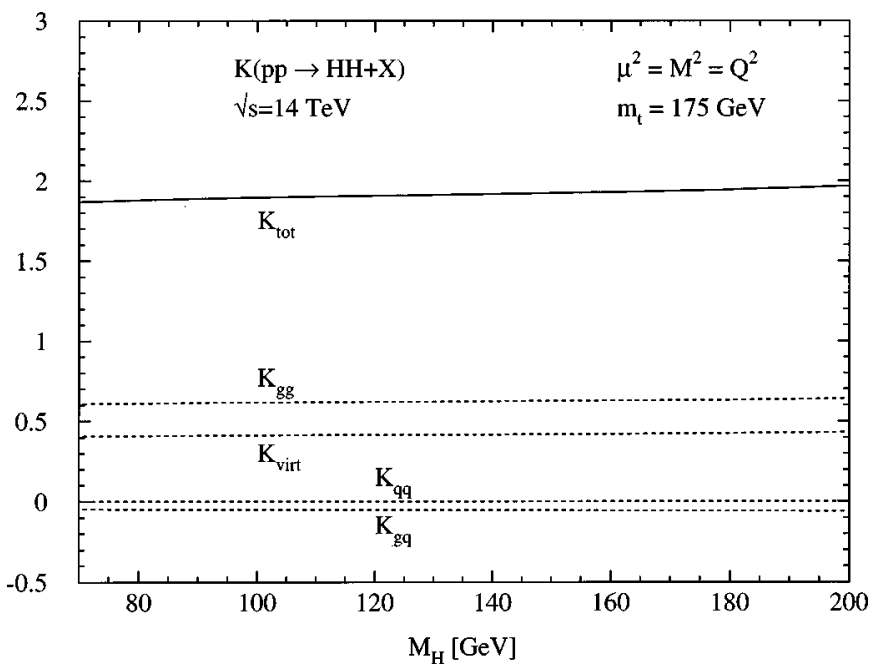

FIG. 6. $K$ factors of the QCD-corrected gluon-fusion SM cross section $\sigma(p p \rightarrow H H+X)$ at the LHC with c.m. energy $\sqrt{s}$ $=14 \mathrm{TeV}$. The dashed lines show the individual contributions of the four terms of the QCD corrections given in Eq. (20).

\section{RESULTS}

\section{A. Standard model}

The analysis for SM Higgs-boson-pair production has been carried out for the LHC c.m. energy $\sqrt{s}=14 \mathrm{TeV}$. The top- and bottom-quark masses have been chosen as $m_{t}$ $=175 \mathrm{GeV}$ and $m_{b}=5 \mathrm{GeV}$. We have adopted the CTEQ4L and CTEQ4M [27] parton densities for the LO and NLO cross sections, respectively, corresponding to the QCD parameters $\Lambda_{5}^{\mathrm{LO}}=181 \mathrm{MeV}$ and $\Lambda_{5}^{\overline{\mathrm{MS}}}=202 \mathrm{MeV}$. Since our results have been obtained in the heavy-quark limit, they can be expected to be reliable for $M_{H} \leqslant 200 \mathrm{GeV}$, based on the experience from single-Higgs-boson production via gluon fusion.

In order to investigate the size of the QCD corrections, we define the $K$ factor $K=\sigma_{\mathrm{NLO}} / \sigma_{\mathrm{LO}}$ as the ratio of the NLO and LO cross sections, where the parton densities and the strong coupling $\alpha_{s}$ are taken at NLO and LO, respectively. The $K$ factor for SM Higgs-boson-pair production is presented in Fig. 6 as a function of the Higgs-boson mass $M_{H}$ and shows little variation with $M_{H}$. It ranges between about 1.9 and 2.0 , thus enhancing the LO cross section significantly. Moreover, the dashed lines of Fig. 6 show the contributions of the individual terms of Eq. (20). It can be clearly inferred that analogously to the case of single-Higgs-boson production the (infrared regularized) virtual and real corrections originating from $g g$ initial states dominate the QCD corrections, while the $g q$ and $q \bar{q}$ initial states do not exceed about $5 \%$ in total. We note that the values shown for $K_{\text {virt }}, K_{g g}, K_{g q}$, and $K_{q q}$ do not exactly add up to $K_{\text {tot }}-1$, since the individual contributions are obtained by taking NLO parton densities and the strong coupling in the NLO cross sections, but LO quantities in the LO cross sections consistently.

In order to investigate the reliability of our results, the residual dependence of the cross section on the renormalization and factorization scales is shown in Fig. 7 for a Higgsboson mass of $M_{H}=200 \mathrm{GeV}$ at LO and NLO. The scale 


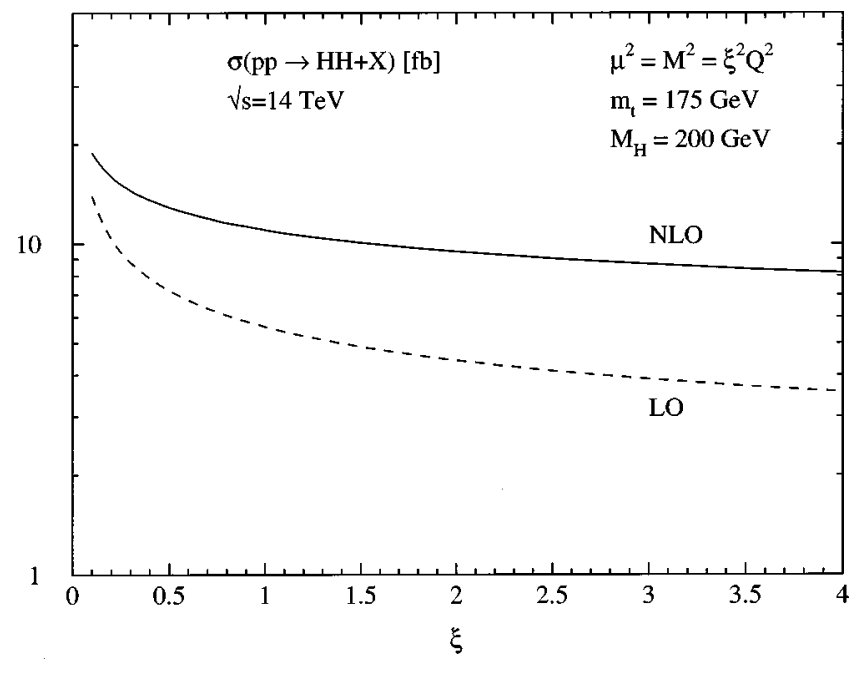

FIG. 7. Renormalization and factorization scale dependence of the SM Higgs-boson-pair-production cross section at LO and NLO for a Higgs-boson mass $M_{H}=200 \mathrm{GeV}$.

dependence significantly decreases compared with the LO result by including the QCD corrections. However, the still monotonic decrease of the NLO cross section with increasing scales signals the need for further improvements. Nevertheless, the theoretical uncertainty of the NLO result can be estimated to about $20 \%$ from the residual scale dependence.

The final results for the total Higgs-boson-pair-production cross sections at LO and NLO are presented in Fig. 8 as a function of the Higgs-boson mass $M_{H}$.

We recall that the full mass dependence of the LO form factors is included in all numerical evaluations; i.e., only the relative NLO correction is treated in the heavy-quark limit, in order to increase the validity of our results. For $M_{H}$ $\$ 200 \mathrm{GeV}$ the cross section exceeds $10 \mathrm{fb}$, leading to more than about 3000 events at the LHC, once the anticipated

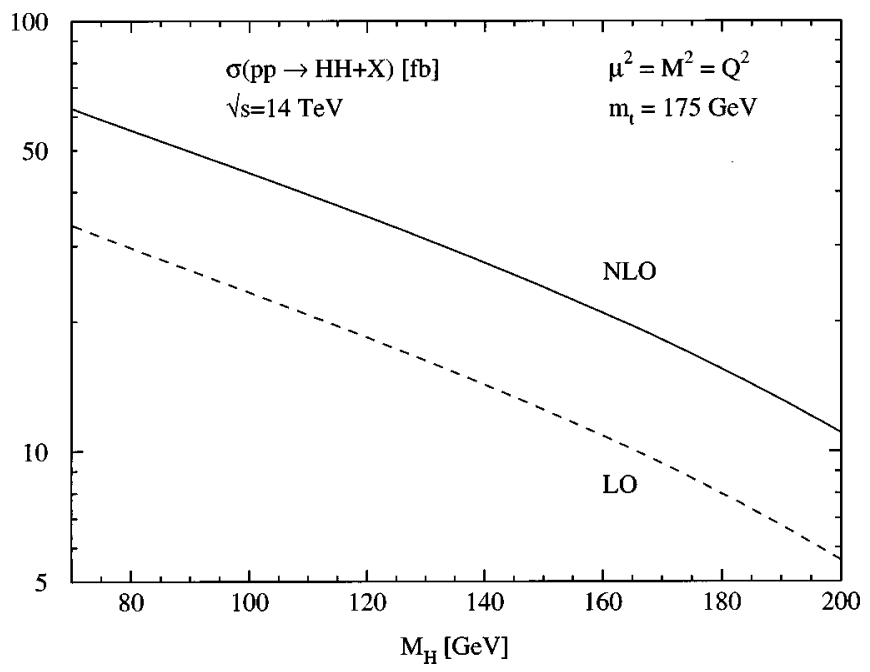

FIG. 8. SM prediction of the Higgs-boson-pair-production cross section at LO and NLO as a function of the Higgs-boson mass $M_{H}$.

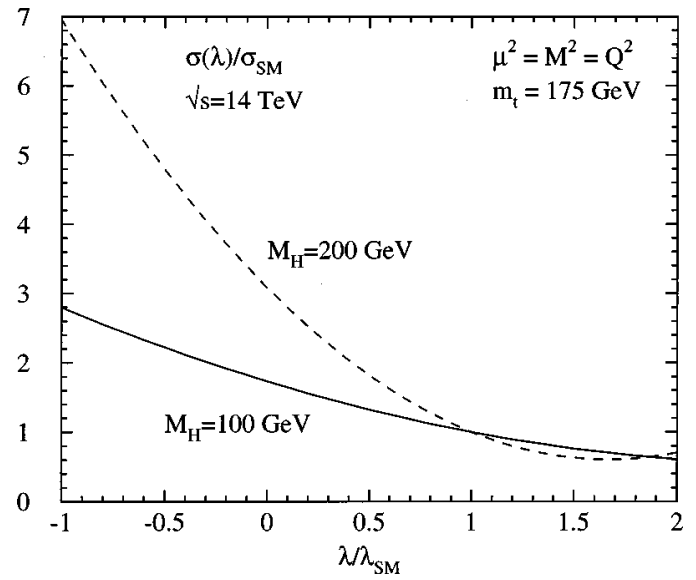

FIG. 9. Ratio of the Higgs-boson-pair-production cross section at NLO with a nonstandard trilinear coupling $\lambda$ and the SM cross section as a function of the trilinear coupling in units of the SM one.

integrated luminosity $\int \mathcal{L} d t=3 \times 10^{5} \mathrm{pb}^{-1}$ is reached. ${ }^{3}$ The typical signatures for Higgs-boson-pair production are $b \bar{b} b \bar{b}$ and $b \bar{b} \tau^{+} \tau^{-}$final states for $M_{H} \lesssim 140 \mathrm{GeV}$. For $M_{H}$ $\gtrsim 160 \mathrm{GeV}$, the Higgs-boson pairs decay predominantly into four vector bosons.

Finally, we present the sensitivity of the SM Higgsboson-pair-production cross section to the trilinear Higgs coupling in Fig. 9, which shows the ratio of the total cross section with a non-standard trilinear coupling $\lambda$ and the SM one as a function of the trilinear coupling, varying in units of the SM coupling. The cross section becomes significantly larger for smaller trilinear couplings, so that this process may serve as a possibility to measure this coupling and test the SM Higgs sector, if the signal can be extracted from the QCD background.

The trilinear Higgs coupling can potentially be measured at high-energy $e^{+} e^{-}$colliders [28]. Since the rate for multiple-Higgs-boson production at $e^{+} e^{-}$colliders is small, high luminosities are required for a sufficient number of events in order to probe this coupling. However, the backgrounds will be well under control.

\section{B. Minimal supersymmetric extension}

Promising MSSM Higgs-boson-pair-production processes at the LHC are $h h, h H, H H, h A$, and $H A$ production with sizable ranges in the parameter space where the cross sections exceed $10 \mathrm{fb}$ [10]. The $g g \rightarrow h h$ process can be used to cover a part of the MSSM parameter space for small $\tan \beta$ via its decay modes into $b \bar{b} \gamma \gamma$ final states [29] in the region where this process is dominated by resonant $g g \rightarrow H \rightarrow h h$ production. Since our calculation is expected to be valid for small values of $\tan \beta$ (where the $b$-quark contribution can be neglected) and Higgs-boson masses below the $t \bar{t}$ threshold, it may be assumed to reliably approximate the cross sections of light-scalar Higgs-boson-pair-production in particular. Gen-

\footnotetext{
${ }^{3}$ The cross section at the Tevatron is less than 0.2 fb for $\sqrt{s}$ $=2 \mathrm{TeV}$.
} 


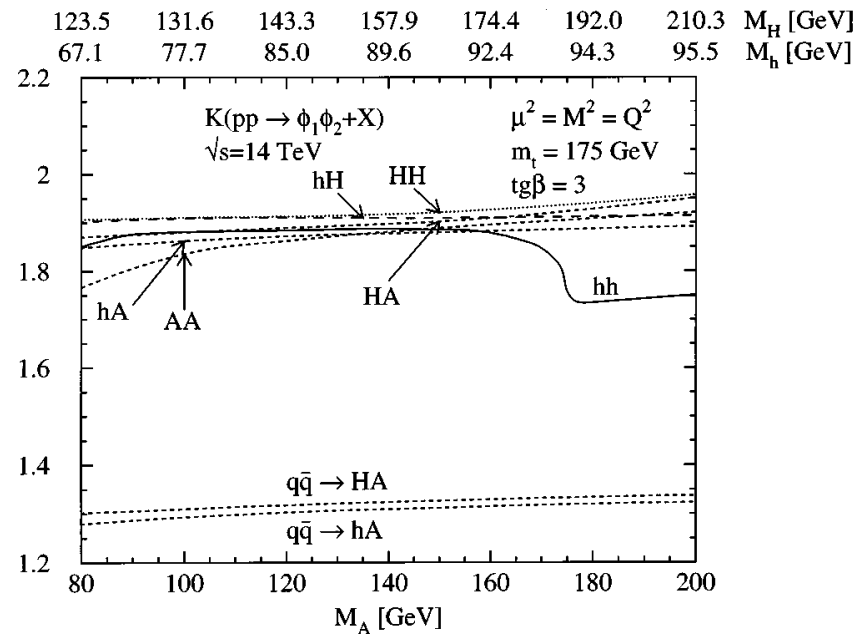

FIG. 10. $K$ factors of the QCD-corrected gluon-fusion and Drell-Yan-like cross sections $\sigma\left(p p \rightarrow \phi_{1} \phi_{2}+X\right)$ at the LHC with c.m. energy $\sqrt{s}=14 \mathrm{TeV}$.

erally, in the MSSM, we expect our results to be valid for $M_{A} \lesssim 200 \mathrm{GeV}$, while for larger pseudoscalar masses at least the heavy $s$-channel Higgs particles become too heavy with respect to the top-quark mass.

In our analysis we have included the MSSM Higgs-boson masses and couplings at the two-loop level in the effectivepotential approach [5]. Moreover, we have included all available higher-order corrections to the total MSSM Higgsboson decay widths [30], which appear in the $s$-channel Higgs propagators of the triangular loop contributions.

The $K$ factors for all Higgs-boson-pair-production processes for $\tan \beta=3$ are presented in Fig. 10 as a function of the pseudoscalar Higgs-boson mass $M_{A}$. The numbers at the top are the corresponding values of the Higgs-boson masses $M_{h}$ and $M_{H}$. The total $K$ factors increase the Drell-Yan-like cross sections by about $30 \%$ and the $g g$ production cross sections by about 60-100\%, thus signaling the importance of the QCD corrections. These are dominated by soft and collinear gluon radiation from $g g$ and $q \bar{q}$ initial states, similar to the SM case discussed above in detail. The sharp decrease of the $K$ factor for $g g \rightarrow h h$ at $M_{A} \sim 175 \mathrm{GeV}$ originates from the resonance contribution $g g \rightarrow H \rightarrow h h$, which is kinematically forbidden below this mass and allowed above. The $K$ factor of resonant single-Higgs-boson production is smaller than the one of continuum $h h$ production.

In spite of the large size of the NLO contributions, the scale dependence significantly decreases, as can be inferred from Fig. 11, which presents the scale dependence of the cross section $\sigma(p p \rightarrow h h+X)$ at LO and NLO for a lightscalar Higgs-boson mass $M_{h}=95.5 \mathrm{GeV}$, corresponding to $M_{A}=200 \mathrm{GeV}$.

Thus the QCD-corrected results turn out to be reliable within about $\pm 20 \%$. The total NLO cross sections for the processes $g g \rightarrow h h, h H, H H$ are presented in Fig. 12 as a function of the pseudoscalar Higgs-boson mass $M_{A}$. The sharp increase of the $h h$ cross section at $M_{A} \sim 175 \mathrm{GeV}$ is due to the fact that the resonant $g g \rightarrow H \rightarrow h h$ process opens up above this mass value, while it is kinematically forbidden below. There are sizable regions where the cross sections

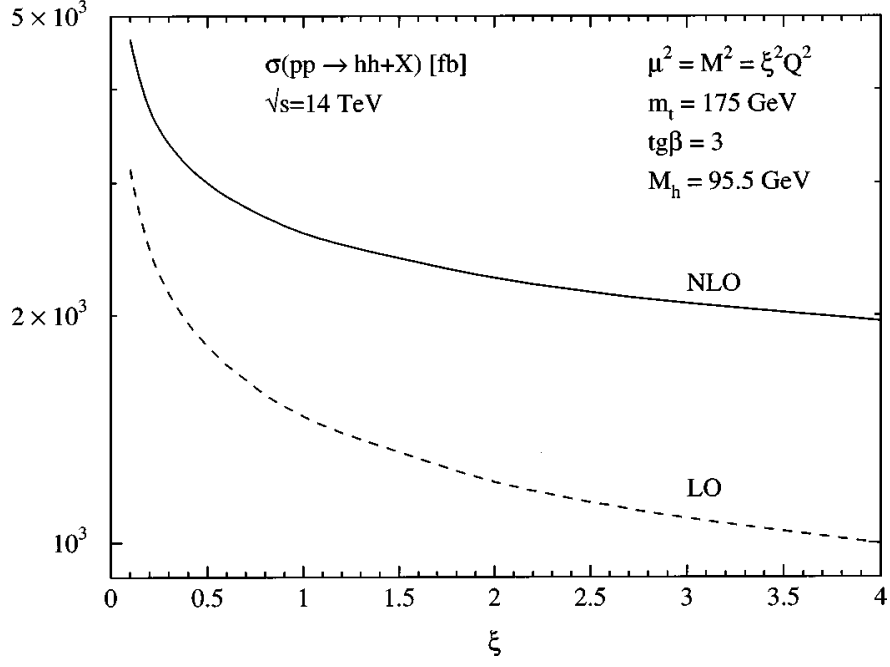

FIG. 11. Renormalization and factorization scale dependence of the Higgs-boson-pair-production cross section $\sigma(p p \rightarrow h h+X)$ at LO and NLO for $\tan \beta=3$ and a Higgs-boson mass $M_{h}$ $=95.5 \mathrm{GeV}\left(M_{A}=200 \mathrm{GeV}\right)$.

exceed a level of $10 \mathrm{fb}$. This is in contrast to $A A$ production, the cross section of which is smaller than $1 \mathrm{fb}$ in the considered parameter space.

The total cross sections for the processes $p p \rightarrow h A, H A$ and their individual contributions from the $g g$ and $q \bar{q}$ initiated processes are presented in Fig. 13 as a function of the pseudoscalar mass $M_{A}$ for $\tan \beta=3$.

While for $H A$-pair production gluon fusion $g g \rightarrow H A$ is always suppressed against the Drell-Yan-like process $q \bar{q}$ $\rightarrow H A$, both corresponding processes are competitive for the light-scalar Higgs particle $h$. Especially for smaller masses $M_{A}$ the total cross sections for $p p \rightarrow h A, H A$ exceed $10 \mathrm{fb}$ and thus potentially provide the possibility to detect these processes. Note, however, that only the gluon-fusion processes are sensitive to the trilinear Higgs couplings.

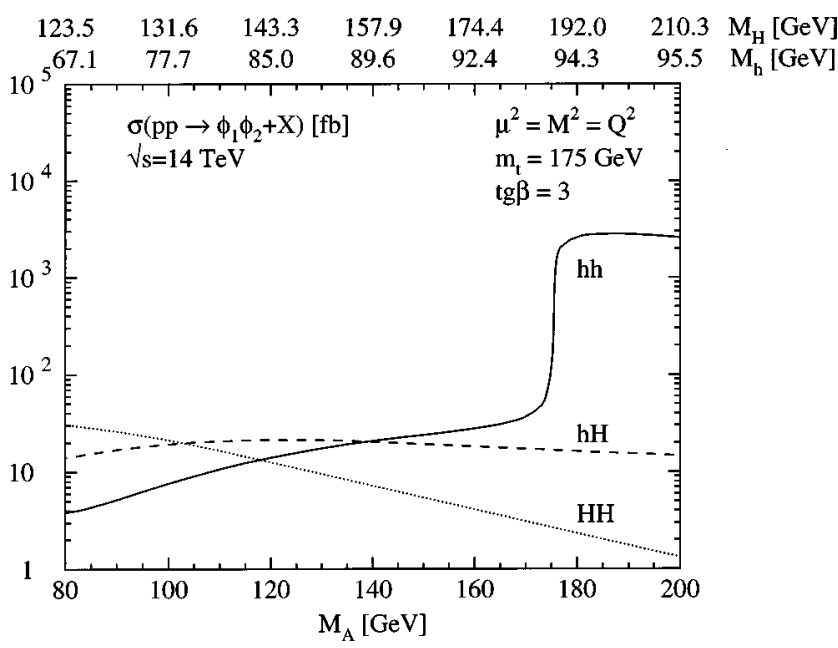

FIG. 12. Scalar Higgs-boson-pair-production cross sections $\sigma(p p \rightarrow h h, h H, H H+X)$ at NLO as functions of the pseudoscalar Higgs-boson mass $M_{A}$ for $\tan \beta=3$. 


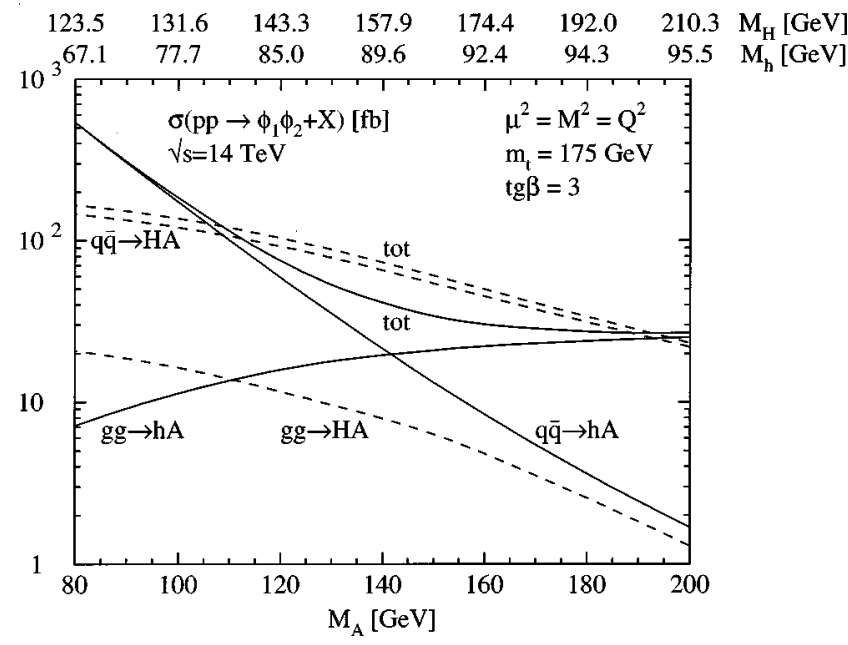

FIG. 13. Scalar and pseudoscalar Higgs-boson-pair-production cross sections $\sigma(p p \rightarrow h A, H A+X)$ at NLO as functions of the pseudoscalar Higgs-boson mass $M_{A}$ for $\tan \beta=3$ and their individual contributions from $g g$ and $q \bar{q}$ collisions. The solid lines correspond to $h A$ and the dashed ones to $H A$ production.

Finally, it should be noted, that in all neutral Higgsboson-pair processes the residual theoretical uncertainties reduce to a level of $20 \%$ after including the QCD corrections.

The corresponding NLO cross section for $h h$ production at the Tevatron with c.m. energy $\sqrt{s}=2 \mathrm{TeV}$ is presented in Fig. 14 as a function of the pseudoscalar mass $M_{A}$. For $M_{A} \gtrsim 175 \mathrm{GeV}$ it exceeds $10 \mathrm{fb}$, since the resonant $g g \rightarrow H$ $\rightarrow h h$ is kinematically possible and dominant in this mass region. The signatures of this process are $b \bar{b} b \bar{b}$ and $b \bar{b} \tau^{+} \tau^{-}$ final states.

Trilinear MSSM Higgs couplings can be measured at high-energy $e^{+} e^{-}$colliders [31], if sufficient numbers of signal events for Higgs-boson-pair production are produced. At the necessary high luminosities, background processes do not cause any problem.

\section{CONCLUSIONS}

We have presented a complete calculation of the two-loop QCD corrections to neutral-Higgs-boson-pair production at the LHC via gluon fusion in the limit of a heavy top quark. This approximation is at least reliable if the invariant mass of the produced Higgs-boson pair is below the $t t$ threshold of the mediating top-quark loops. We have analyzed the results within the SM and MSSM and found large increases of the cross sections by about 60-100\%. The QCD corrections to the associated production processes $q \bar{q} \rightarrow h A, H A$ coincide

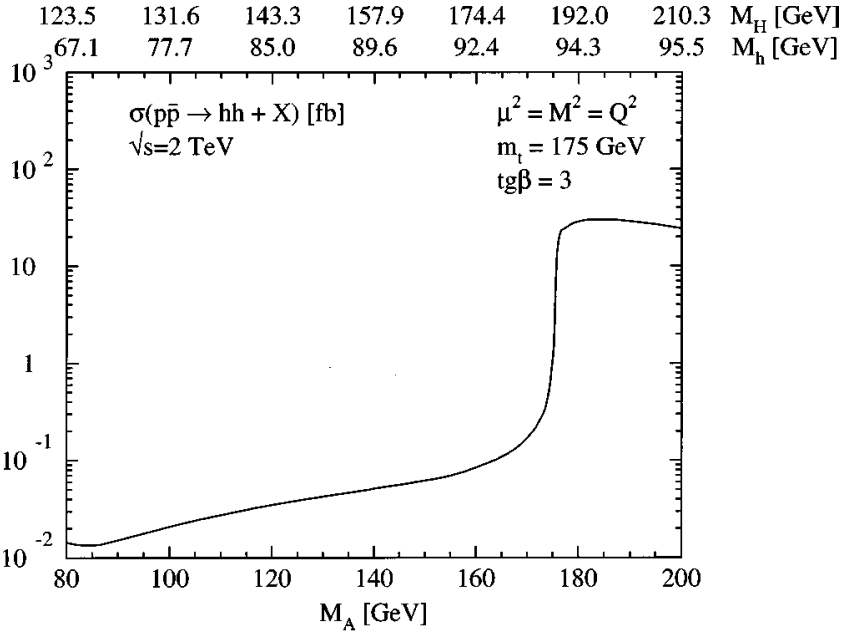

FIG. 14. Scalar Higgs-boson-pair-production cross section $\sigma(p \bar{p} \rightarrow h h+X)$ at NLO as a function of the pseudoscalar Higgsboson mass $M_{A}$ for $\tan \beta=3$ at the Tevatron $p \bar{p}$ collider with c.m. energy $\sqrt{s}=2 \mathrm{TeV}$.

with those to the Drell-Yan process $q \bar{q} \rightarrow Z^{*}$, thus increasing the cross sections by about $30 \%$. The QCD corrections stabilize the theoretical predictions compared with the LO results, which exhibit large theoretical uncertainties. After including the QCD corrections, the remaining theoretical uncertainties are reduced to a level of about $20 \%$.

Except for $A A$-pair production, all Higgs-boson-pairproduction cross sections of the SM and MSSM exceed $10 \mathrm{fb}$ at LHC energies in certain regions of the parameter spaces. As soon as the $H \rightarrow h h$ channel opens, the cross section for $p p \rightarrow h h+X$ even reaches $10^{3} \mathrm{fb}$ at the LHC and is still larger than $10 \mathrm{fb}$ at the Tevatron for $\tan \beta=3$.

Moreover, we have shown that the NLO prediction of Higgs-boson-pair production is sensitive to a deviation of the trilinear Higgs coupling from its SM value, rendering this process well suited for studying this coupling in $p p$ collisions.

\section{ACKNOWLEDGMENTS}

We would like to thank T. Plehn and P. M. Zerwas for useful discussions. Moreover, we are grateful to P. M. Zerwas for reading the manuscript and his valuable comments. BNL is supported by U.S. Department of Energy Contract No. DE-AC02-98CH10886. The Institut Für Theoretische Physik is supported by Bundesministerium für Bildung und Forschung (BMBF), Bonn, Germany, under Contract No. 05 $7 \mathrm{HH}$ 92P (5), and by the EU: Program Human Capital and Mobility through Network Physics at High Energy Colliders under Contract No. CHRX-CT93-0357 (DG12 COMA).
[1] P. W. Higgs, Phys. Lett. 12, 132 (1964); Phys. Rev. 145, 1156 (1966); F. Englert and R. Brout, Phys. Rev. Lett. 13, 321 (1964); G. S. Guralnik, C. R. Hagen, and T. W. Kibble, ibid. 13, 585 (1964).
[2] For reviews on the Higgs sector in the standard model and in its supersymmetric extensions, see J. F. Gunion, H. E. Haber, G. L. Kane, and S. Dawson, The Higgs Hunter's Guide (Addison-Wesley, Reading, MA, 1990); S. Dawson, in "Per- 
spectives in Higgs Physics,' edited by G. Kane, Report No. BNL-HET-SD-97-004, hep-ph/9703387; M. Spira and P. M. Zerwas, presented at the 36th Internationale Universitätswochen für Kernphysik und Teilchenphysik, Schladming 1997, Report No. CERN-TH/97-379, hep-ph/9803257.

[3] Y. Okada, M. Yamaguchi, and T. Yanagida, Prog. Theor. Phys. 85, 1 (1991); H. E. Haber and R. Hempfling, Phys. Rev. Lett. 66, 1815 (1991); J. Ellis, G. Ridolfi, and F. Zwirner, Phys. Lett. B 257, 83 (1991).

[4] R. Hempfling and A. Hoang, Phys. Lett. B 331, 99 (1994); S. Heinemeyer, W. Hollik, and G. Weiglein, Report No. KA-TP2-1998, hep-ph/9803277.

[5] M. Carena, J. R. Espinosa, M. Quirós, and C. E. M. Wagner, Phys. Lett. B 355, 209 (1995); M. Carena, M. Quirós, and C. E. M. Wagner, Nucl. Phys. B461, 407 (1996).

[6] V. Barger, T. Han, and R. J. N. Phillips, Phys. Rev. D 38, 2766 (1988).

[7] D. A. Dicus, K. J. Kallianpur, and S. S. D. Willenbrock, Phys. Lett. B 200, 187 (1988); A. Abbasabadi, W. W. Repko, D. A. Dicus, and R. Vega, Phys. Rev. D 38, 2770 (1988); Phys. Lett. B 213, 386 (1988).

[8] K. J. Kallianpur, Phys. Lett. B 215, 392 (1988).

[9] E. W. N. Glover and J. J. van der Bij, Nucl. Phys. B309, 282 (1988).

[10] T. Plehn, M. Spira, and P. M. Zerwas, Nucl. Phys. B479, 46 (1996).

[11] A. Djouadi, presented at the Workshop Theory of LHC Processes, CERN, Geneva, 1998.

[12] M. Krämer, E. Laenen, and M. Spira, Nucl. Phys. B511, 523 (1998)

[13] A. Djouadi, M. Spira, and P. M. Zerwas, Phys. Lett. B 264, 440 (1991); S. Dawson, Nucl. Phys. B359, 283 (1991).

[14] M. Spira, A. Djouadi, D. Graudenz, and P. M. Zerwas, Nucl. Phys. B453, 17 (1995).

[15] J. Ellis, M. K. Gaillard, and D. V. Nanopoulos, Nucl. Phys. B106, 292 (1976); A. I. Vainshtein, M. B. Voloshin, V. I. Zakharov, and M. A. Shifman, Sov. J. Nucl. Phys. 30, 711 (1979).

[16] B. A. Kniehl and M. Spira, Z. Phys. C 69, 77 (1995).

[17] S. L. Adler, Phys. Rev. 177, 2426 (1969); J. S. Bell and R.
Jackiw, Nuovo Cimento A 60, 47 (1969).

[18] A. Djouadi, M. Spira, and P. M. Zerwas, Phys. Lett. B 311, 255 (1993); M. Spira, A. Djouadi, D. Graudenz, and P. M. Zerwas, ibid. 318, 347 (1993); R. P. Kauffman and W. Schaffer, Phys. Rev. D 49, 551 (1994).

[19] S. L. Adler and W. A. Bardeen, Phys. Rev. 182, 1517 (1969); R. Jackiw, Lectures on Current Algebra and its Applications (Princeton University Press, Princeton, 1972).

[20] G. Altarelli and G. Parisi, Nucl. Phys. B126, 298 (1977).

[21] J. Küblbeck, M. Böhm, and A. Denner, Comput. Phys. Commun. 60, 165 (1990); H. Eck and J. Küblbeck, "Guide to FeynArts 1.0," report, University of Würzburg, 1992.

[22] V. A. Smirnov, Phys. Lett. B 394, 205 (1997).

[23] S. G. Gorishny, Nucl. Phys. B319, 633 (1989); V. A. Smirnov, Commun. Math. Phys. 134, 109 (1990); Renormalization and Asymptotic Expansions (Birkhäuser-Verlag, Basel, 1991); F. V. Tkachov, Int. J. Mod. Phys. A 8, 2047 (1993); G. B. Pivovarov and F. V. Tkachov, ibid. 8, 2241 (1993).

[24] S. Wolfram, Mathematica-A System for Doing Mathematics by Computer (Addison-Wesley, Redwood City, CA, 1988).

[25] G. 't Hooft and M. Veltman, Nucl. Phys. B44, 189 (1972); P. Breitenlohner and D. Maison, Commun. Math. Phys. 52, 11 (1977).

[26] W. Furmanski and R. Petronzio, Z. Phys. C 11, 293 (1982) and references therein.

[27] H. L. Lai, J. Huston, S. Kuhlmann, F. Olness, J. Owens, D. Soper, W. K. Tung, and H. Weerts, Phys. Rev. D 55, 1280 (1997).

[28] G. J. Gounaris, D. Schildknecht, and F. M. Renard, Phys. Lett. 83B, 191 (1979); 89B, 437(E) (1980); J. F. Gunion, L. Roszkowski, A. Turski, H. E. Haber, G. Gamberini, B. Kayser, S. F. Novaes, F. Olness, and J. Wudka, Phys. Rev. D 38, 3444 (1988); V. Barger and T. Han, Mod. Phys. Lett. A 5, 667 (1990); F. Boudjema and E. Chopin, Z. Phys. C 73, 85 (1996).

[29] E. Richter-Was, D. Froidevaux, F. Gianotti, L. Poggioli, D. Cavalli, and S. Resconi, Report No. CERN-TH/96-111.

[30] For recent reviews, see, e.g., A. Djouadi, Int. J. Mod. Phys. A 10, 1 (1995); M. Spira, Fortschr. Phys. 46, 203 (1998).

[31] A. Djouadi, H. E. Haber, and P. M. Zerwas, Phys. Lett. B 375, 203 (1996) 